\title{
Fast Reservation List-to-Send MAC Protocol in Wireless Networks
}

\author{
Yayan Li and Kai Liu \\ School of Electronics and Information Engineering \\ Beihang University, Beijing, 100191, China \\ Email: liyangyayan@yahoo.com.cn, liuk@buaa.edu.cn
}

\begin{abstract}
Fast reservation list-to-send (FRLS) medium access control (MAC) protocol is proposed to apply to wireless networks with long propagation delay. In the protocol, a central control node $(\mathrm{CCN})$ is applied to allocate the channel resources of two channels, and all the nodes transmit packets over the two channels without collision. Packet train transmission mechanism ensures that a number of packets are transmitted continuously with small guard interval and just a one-way propagation delay. Once a node successfully reserves the channel resources for a communication session, it can transmit the whole message without the need of any other reservations, which gain high utilization of the channels. Moreover, $\mathrm{CCN}$ can provide uniform time benchmark to make the network working in an asynchronous fashion. Simulation results show that FRLS protocol outperforms centralized scheduling-based MAC (CSMAC) protocol in terms of throughput, average packet access delay and average packet dropping rate, and effectively resolves low channel utilization problem due to long propagation delay.
\end{abstract}

Keywords-wireless network; medium access control; reservation; collision-free transmission; long propagation delay

\section{INTRODUCTION}

Medium access control protocol solves the problem that multiple users or multiple nodes share the channel resources to transmit the traffic packets. A better MAC protocol must make sure that it has relatively higher channel throughput, lower packet access delay, and the fairness of using the channel resources. Besides, we need to consider the stability and complexity of the protocol in the process of its design.

MAC protocol always can be divided into three categories, i.e., fixed allocation, random allocation and on-demand allocation [1]. Fixed allocation divides one channel into a number of mutually independent sub-channels. In the communication process, one of the sub-channels will be allocated to each user. The typical examples are FDMA, TDMA and CDMA. It provides with reliable service and it applies to the network with higher traffic continuity and real-time requirement. For burst traffic or under the circumstances the nodes do not transmit packets for a long time, fixed allocation will incur large waste of channel resources.

Random allocation is the kind of access control that all

This work was supported by NSF for Innovative Research Groups of China under Grant 60921001, the National Outstanding Youth Science Foundation of China under Grant 60625102, NSBS Program of BUAA, China under Grant 221235, and the National 863 Program of China under Grant 2006AA12A103.

CHINACOM 2010, August 25-27, Beijing, China

Copyright (C) 2011 ICST 973-963-9799-97-4

DOI 10.4108/chinacom.2010.10 the nodes sense the channel to decide whether it is being used or free. If the channel is free, the nodes with packets to send can obtain the right to use the channel to transmit the data in random time delay. ALOHA [2] protocol series and CSMA [3] [4] protocol series are all random allocation protocols. This kind of access method applies to deal with burst traffic or small traffic load network. In this case, it can achieve shorter access delay and higher channel utilization. Besides, random allocation resolves channel resources waste problem. However, it incurs packets collision problems caused by exposed terminal, hidden terminal and intrusion terminal. Thus, the average packet delay gets longer, channel throughput decreases and the performance of access gets worse when there are heavy packets collisions in the network.

On-demand allocation is also called reservation access method or non-competitive method such as PRMA and DAMA [5]. An example of the on-demand allocation is polling mechanism. The station polls every node according to certain cycle sequence to see whether they have packets to transmit. If the node being polled has packet to transmit, the node has the right to access to the channel to transmit its packet as soon as possible. Otherwise, it polls another node. Non-competitive method resolves the problem of channel resources wasting and the long access delay caused by contention. However, if there is small traffic load in the network, polling mechanism will increase the overhead and induce lower channel resources utilization.

A good MAC protocol is generally a combination of random allocation and on-demand allocation.

In this paper, we propose a MAC protocol for wireless networks, especially for the network with long propagation delay. The proposed protocol allows network to transmit a number of packets derived from different nodes continuously during a transmission period. So we call the protocol fast reservation list-to-send multiple access protocol. If there are multiple handshakes in a packet transmission period, propagation delay will have a greater impact on making good use of the limited channel resources, especially in the aeronautical communication network, satellite communication network and deep space communication network. The proposed FRLS protocol will solve the long propagation delay problem.

The rest of the paper is outlined as follows. In section 
II, the network model of FRLS protocol is described. In Section III, the details of the FRLS protocol is discussed. The performance evaluation is carried out in Section IV and an extended set of results for networks with different parameters is presented. Section V draws a conclusion of the paper.

\section{NeTWORK MODEL}

The network consists of a central control node and a number of neighbor nodes. The central control node has a full-duplex transceiver. The rest of the nodes each only have a half-duplex transceiver. There are two traffic channels to transmit packets in the network. Every node in the network is just one-hop or two-hop distance between each other. If the nodes are multi-hop distance between each other, clustering approach [7] will be used to group nodes to clusters. A cluster is a subset of nodes which can communicate with a cluster head and with each other. $\mathrm{CCN}$ located at the center of the network and it is neighbor node of any other nodes and can directly communicate with them. The source node's traffic packets are transmitted to the destination node with the help of CCN. Neighbor nodes can't communicate with each other directly. As CCN broadcasts start reservation (SR) signal which can provide a coarse synchronization to the network, allowing network working in asynchronous transfer mode. CCN and neighbor nodes transmit traffic packets simultaneously in two different channels and collision will not occur. Neighbor nodes are always in the second channel to listen or to receive the packets coming from $\mathrm{CCN}$ except when they are in the period of reservation or transmitting their own traffic packets in the first channel.

The time slots that CCN distributes to the neighbor nodes with packets to send include transmitting time, transceiver conversion time and necessary time interval to avoid disturbance. Channel overhead mainly comes from traffic packet propagation delay, the transmission time of SR signal, transmission time of access reservation (AR) packet and access result distribution time (AD). Relative to the long propagation delay, signal processing delay can be ignored. As there is a CCN to allocate the time slots, channel resources can be full use of and avoid collision. Without taking channel error into consideration, packet-dropping rate is zero in the process of transmission. The packet-dropping is caused by heavy traffic load. As a result, traffic packet has to be discarded in a long time delay.

\section{FRLS PROTOCOL}

In FRLS protocol, CCN takes on the advantage of transmitting and receiving in different channel simultaneously and it coordinates the competitive access and traffic packet transmission of neighbor nodes, so the protocol avoid the adverse effect of low throughput resulting from long propagation delay.

Fig.1 shows the timing diagram for the FRLS protocol.
In the beginning of a frame, $\mathrm{CCN}$ sets up the number of contention access reservation time slot $\left(N_{A R}\right)$ based on the current number of success access nodes and free channel resources. The details of the protocol are as follows.

If there are free channel resources, then set $N_{A R}=\max \left(4, n^{*} N_{\text {fire }}\right)$. Otherwise set $N_{A R}=0$, do not have to reserve and transmit packets without collision directly. $N_{\text {free }}=N_{p k t}-N_{p k t \_n o w} . N_{\text {free }}, N_{\text {plt }}$ and $N_{\text {pkt_now }}$ denote the number of free transmission time slots in the current frame, the largest number of transmission time slots in a frame and the number of nodes successful access to the channel before the new period of reservation. The symbol $n$ represents a coefficient which can be set according to the specific requirements of the network. The value $\mathrm{n}$ ranges from 2 to 5 . The specific value of $n$ is based on the reservation collision. If the collision is heavy, increase the value of $\mathrm{n}$. Otherwise, lower the value of $\mathrm{n}$. The aim of changing the value of $\mathrm{n}$ is to improve channel throughput without severely affecting the probability of competitive reservation success.

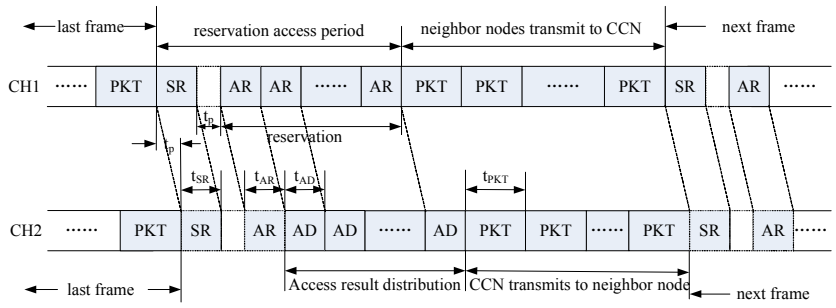

Fig. 1. FRLS protocol timing diagram.

After setting up $N_{A R}, \mathrm{CCN}$ transmits SR signal to all of the nodes in the first channel to inform them of starting competitive reservation. Next, after the propagation delay $t_{p}$, nodes receive the SR packet in the first channel $\left(\mathrm{CH}_{1}\right)$ and the nodes with traffic packets to send choose one of the AR packets randomly to send reservation signal to $\mathrm{CCN}$ in $\mathrm{CH}_{1}$ to reserve the channel resources. Following the transmission of AR packets, all of the nodes turn to the second channel $\left(\mathrm{CH}_{2}\right)$ to receive the result of reservation. $\mathrm{CCN}$ announces the results by sending $\mathrm{AD}$ packets to the reservation nodes after having received AR packets from neighbor nodes. If the node reserves successfully, $\mathrm{CCN}$ will allocate channel resources to the node. If fails, CCN notifies it to reserve in the next frame and the failed nodes will turn to $\mathrm{CH}_{1}$ to sense the signal from $\mathrm{CCN}$. All of the information is carried by $\mathrm{AD}$ packets. The successful nodes still stay in $\mathrm{CH}_{2}$ to receive the traffic packets transmitted by $\mathrm{CCN}$ and get ready to turn to $\mathrm{CH}_{1}$ to transmit its own traffic packets when the allocated time slot comes. Before neighbor node transmits its own traffic packets, we need to judge whether all of the packets of the node have been transmitted. If so, the node transmits a packet to notify $\mathrm{CCN}$ and $\mathrm{CCN}$ will not allocate channel resources to it any more. Otherwise, the node transmits its traffic packet by using the allocated channel resources. After transmitting the traffic packet, the node turns to $\mathrm{CH}_{2}$ to receive CCN's packets. Finally, judge 
whether the frame is finish. If not, the next successful reservation node will transmit its packet following the former successful node. Otherwise, all of the nodes turn to $\mathrm{CH}_{1}$ to sense the signal from $\mathrm{CCN}$ and the next frame starts.

In the protocol, the node can choose AR time slot randomly with the same possibility or according to certain probability distribution. We take random selection method in the following simulation. There is an issue need to be taken into consideration. When CCN transmits traffic packet to a node in $\mathrm{CH}_{2}$, however, the right node is transmitting its packet in $\mathrm{CH}_{1}$. This case needs to be avoided. If not, the node can't receive CCN's packet successfully. This problem can be solved under CCN's coordination. Besides, for the nodes that has reserved successfully and not all of its current traffic packets have been transmitted, they do not have to reserve in the next frame and they use the former channel resources allocated by $\mathrm{CCN}$. As AR packet is short and set up according to the free channel resources, the overhead is low and the channel throughput is high. According to the protocol, the throughput of $\mathrm{CH}_{1}$ and $\mathrm{CH}_{2}$ are as follows.

$$
\begin{aligned}
\mathrm{S}_{C H 1} & =\frac{N_{p k t} \times t_{p k t} \times R_{b}}{t_{S R}+t_{p}+N_{A R} \times t_{A R}+N_{p k t} \times t_{p k t}} \\
\mathrm{~S}_{C H 2} & =\frac{N_{p k t}^{\prime} \times t_{p k t} \times R_{b}}{t_{S R}+t_{p}+N_{A D} \times t_{A D}+N_{p k t}^{\prime} \times t_{p k t}}
\end{aligned}
$$

The symbols $N_{p k t}, N_{p k t}, t_{S R}, t_{A R}, t_{p k t}, t_{A D}, R_{b}$, denote the largest number of traffic packets which can be transmitted in a frame in $\mathrm{CH}_{1}$, the largest number of traffic packets which can be transmitted in a frame in $\mathrm{CH}_{2}$, a SR packet transmission time, a AR packet transmission time, a traffic packet transmission time, a $\mathrm{AD}$ packet transmission time and the date rate or the channel.

FRLS protocol is the combination of random allocation and non-competitive allocation. It overcomes the low channel throughput and long access delay problem caused by long propagation delay. First, the protocol use random allocation method to send a short AR packet to reserve the right to use the channel. In the process of reservation, AR packets collision is possible to occur. But the collision only happens in the AR packets rather than data packets. If the reservation successes, $\mathrm{CCN}$ allocates channel resources to the successful nodes, then the traffic packets will be transmitted without collision. There are not exposed terminal, hidden terminal and intrusion terminal issues in FRLS protocol. The proposed protocol has merits of easy handshaking mechanism, low control overhead, high channel throughput and short access delay.

\section{PERFormance EVAluation}

In this paper, we use $\mathrm{C}$ language to simulate and evaluate the performance of FRLS protocol. In order to verify the validity of FRLS protocol to solve the problem of long propagation delay, we will compare FRLS protocol with
CSMAC protocol which is newly proposed in literature [6]. We mainly consider these three performances under different conditions: channel throughput, average packet access delay and average packet dropping rate. The so called channel throughput in the paper represents the amount of information transmitted per unit time. We mainly discuss the impact of the largest number of packets in a message $\left(P_{\max }\right), N_{p k t}$ and the length of packet $\left(L_{p k t}\right)$ on FRLS protocol in the following simulation.

\section{A. Simulation Environment}

All of the neighbor nodes in the network randomly distribute in a radius of $1 \mathrm{~km}$ area and the central control node locates in the center of the region. The communication radius is $1 \mathrm{~km}$. So, all the neighbor nodes in the network are just one-hop or two-hop distance between each other. In the simulation, we use satellite communication network as the simulation context to set up the parameters. In satellite communication network, propagation delay can be about $2.5 \mathrm{~ms}$. Each station in the network communicates directly with the CCN at a data rate $\left(R_{b}\right)$ of $1 \mathrm{Mbps}$. There are two channels in FRLS protocol, but just one channel in CSMAC protocol. In order to ensure the overall data rate of the two protocols equaling to each other, we set date rate of each channel of FRLS protocol to be $0.5 \mathrm{Mbit} / \mathrm{s}$. Other parameters are set as follows. $t_{p}=2.5 \mathrm{~ms}, N_{p k t}=N_{p k t}=20, t_{p k t}=1 \mathrm{~ms}$, $t_{S R}=t_{A R}=t_{A D}=0.02 \mathrm{~ms}, P_{\max }=5$. The traffic packets of each node are generated by Poisson stochastic process. The number of packets in each message is stochastic integer ranging from 1 to $P_{\max }$.

\section{B. The Impact of Different $P_{\max }$ on Network Performance}

Fig.2, Fig. 3 and Fig. 4 show the impact of different $P_{\max }$ on network performance of FRLS protocol. In the simulation run, we set $P_{\max }$ to 1,3 and 5. There are 50 nodes in the network, and packet length is set to $1 \mathrm{kbits}$. Then we get the simulation results as the figures mentioned above showed. We can see that FRLS protocol far outperforms CSMAC protocol in throughput and average packet dropping rate. And when $P_{\max }$ is larger than 1, average packet access delay of FRLS protocol is smaller than CSMAC protocol's. Furthermore, with the increase of $P_{\max }$, FRLS protocol's throughput decreases when the traffic load has not saturated, but slightly increases when the traffic load has saturated. Average packet access delay decreases with the increase of $P_{\max }$. Because of FRLS protocol's list-to-send mechanism, nodes can transmit 20 packets with just one reservation period and a one-way propagation delay in a transmission period. And once a node reserves the channel successful, it can transmit all of its packet trains without a second reservation. However, CSMAC protocol adopts binary exponential backoff mechanism and every time it transmits a packet, there will be a propagation delay. So, FRLS protocol well solves long propagation delay problem and gets high throughput and small access delay. Especially, with the increase of $P_{\max }$, the advantage of FRLS protocol to solve propagation delay problem is more obvious. To make sure 
the traffic load equal to each other in different $P_{\max }$, we adjust the arrival rate $\mathrm{f}$ every node. If $P_{\max }$ is smaller, there will be more packets to send in every node and throughput will increase when traffic load is under saturation. It is because that there are more free channel resources used to transmit data and the utilization of the channel increases. Moreover, with the control of $\mathrm{CCN}$, there is not collision in the transmission period of FRLS protocol. But CSMAC protocol is not a collision free protocol. So, FRLS protocol outperforms CSMAC protocol in average packet dropping rate. Through the analysis, we can say that if the $P_{\max }$ is set to a proper value, FRLS protocol can get a better performance in the application.

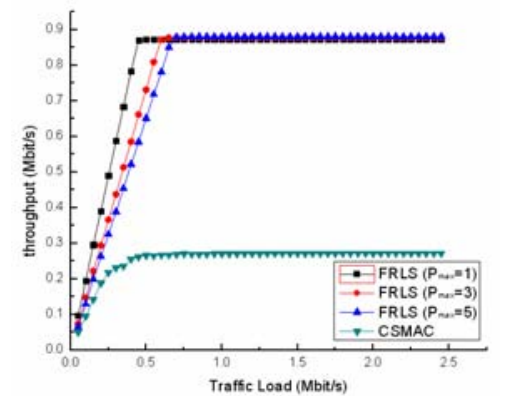

Fig. 2. Average packet access delay with different $P_{\max }$.

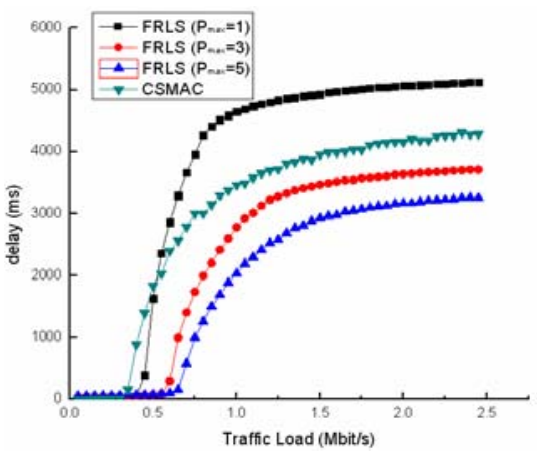

Fig. 3. Throughput with different $P_{\max }$.

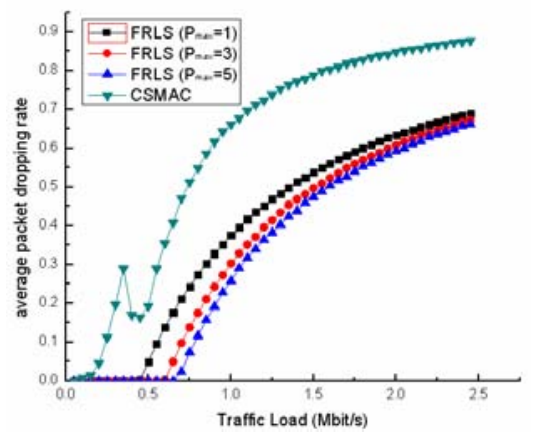

Fig. 4. Average packet dropping rate with different $P_{\max }$.

\section{The Impact of Different $N_{\text {pkt }}$ on Network Performance}

We set the number of the nodes to be 50 and $N_{p k t}$ to be $10,15,20$ and 25 . The simulation results are shown in Fig.5, Fig.6 and Fig.7. We can see that FRLS protocol far outperforms CSMAC protocol in throughput, access delay and average packet dropping rate, no matter what the value of $N_{p k t}$ is. It can be seen from Fig. 5 that $N_{p k t}$ hardly effects channel throughput and average packet access delay when traffic load has not saturated. But when traffic load get larger, throughput increases and average packet delay decreases with the increase of $N_{p k t}$. It is because that the throughput FRSL MAC protocol is mainly affected by propagation delay when traffic load is heavy. If there are more packets sent in a frame, the proportion of propagation delay is smaller in the frame. So the throughput becomes higher. Fig.6 shows that $N_{p k t}$ have little effect on average access delay when the traffic load is low. It is because that all packets are generated evenly in time and they are able to quickly and effectively contend channel resources at different time period. When traffic load increases, not all of the packets can effectively get the right to access to channel successfully. With the increase of $N_{p k t}$, more nodes can use the channel resources to transmit the packets in a frame and fewer nodes need to wait for the next frame to contend the channel resources. So, traffic packets can be sent timely and average packet access delay becomes lower. But, if $N_{p k t}$ is large enough, the difference will be subtle.

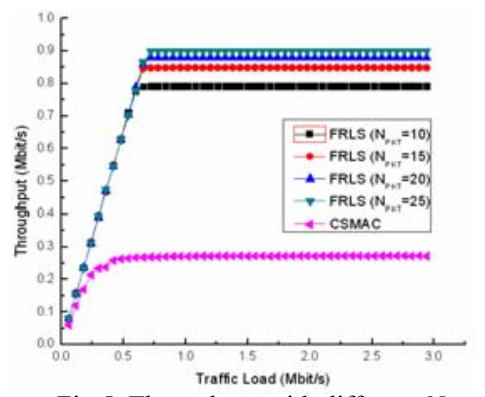

Fig 5. Throughput with different $N_{p k t}$.

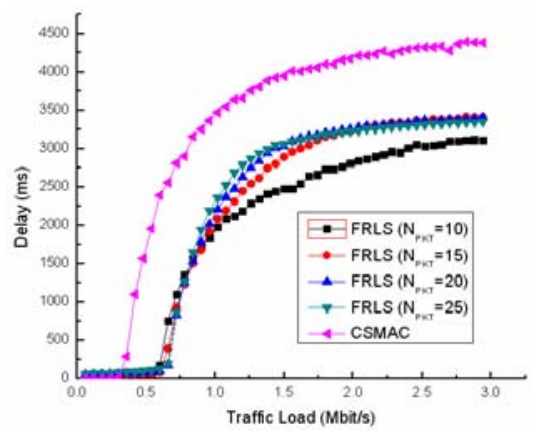

Fig. 6. Average packet delay with different $N_{p k t}$.

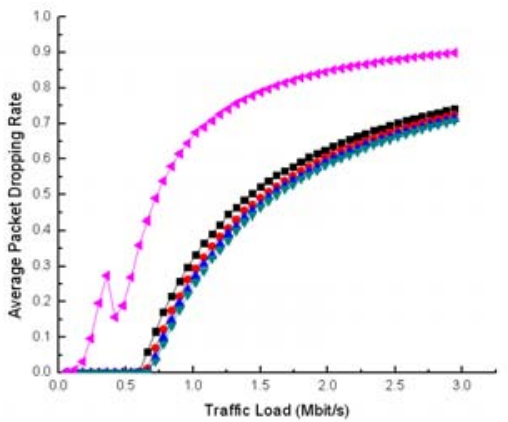

Fig. 7. Average packet dropping rate with different $N_{p k t}$. 


\section{The Impact of $L_{p k t}$ on Network Performance}

It can be seen from Fig.8, Fig.9 and Fig.10 that channel throughput gets higher, average packet access delay gets longer and average packet dropping rate gets smaller when packet length $\left(L_{p k t}\right)$ gets longer in FRLS protocol. However, the average packet access delay of CSMAC protocol decreases with the increase of packet length. When $L_{p k t}$ is smaller than 1.3kbits, FRLS protocol outperforms CSMAC protocol in both throughput and delay. With the increasing of the traffic packet length, the proportion of valid date in a frame will get larger and the negative impact on channel throughput coming from reservation overhead and long propagation delay will become smaller. So the throughput gets higher. However, longer traffic packets, which means the length of each frame gets larger, will lead to the nodes with packets to send but having not yet access successfully to the channel needs to wait longer to contend to access in the next frame. As a result, average packet access delay of FRLS protocol gets larger. To CSMAC protocol, it will not be affected by list-to-send mechanism. The most important factors that affect delay are propagation delay and packets arrival rate. With the increase of $L_{p k t}$, both the proportion of propagation delay in a frame and packets arrival rate decreases. Collision rate will decrease at the same time. Thus, average packet access delay of CSMAC protocol decreases.

\section{CONCLUSION}

A MAC protocol is proposed for wireless network with long propagation delay such as satellite communication network, aeronautical communication network and deep space communication network in the paper. In the control of CCN, the network can work in an asynchronous fashion. The key idea is that there are two channels to transmit a number of packets in a frame with random reservation. Easy handshaking method and packet train transmission mechanism efficiently decrease the negative impact on channel throughput caused by long propagation delay. Besides, the average packet access delay is short and average packet dropping rate is low due to collision free. The protocol resolves the problem of the negative impact on the performance of network caused by long propagation delay.

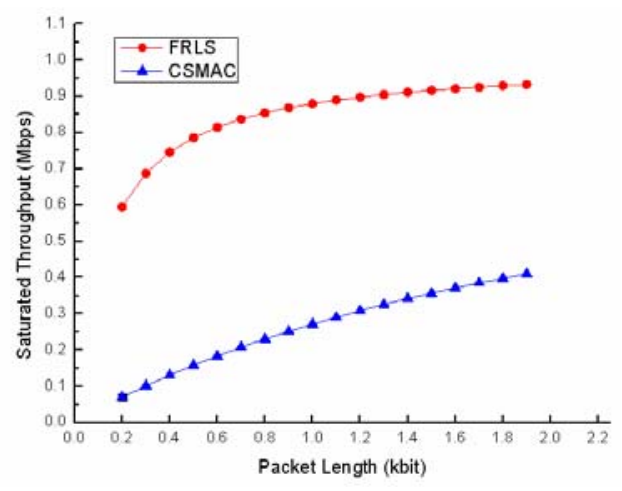

Fig. 8. Saturated throughput with different $L_{p k t}$.

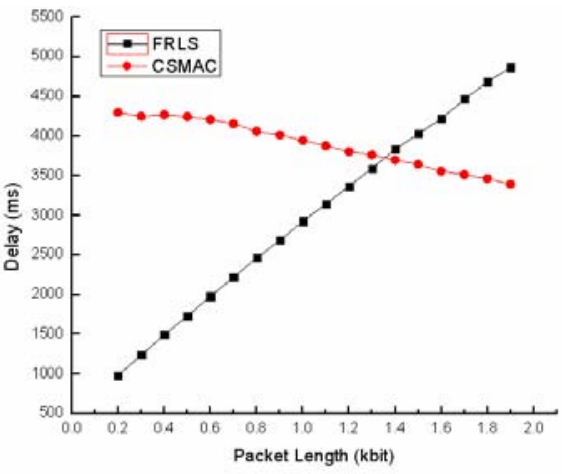

Fig. 9. Average packet access delay with different $L_{p k t}$.

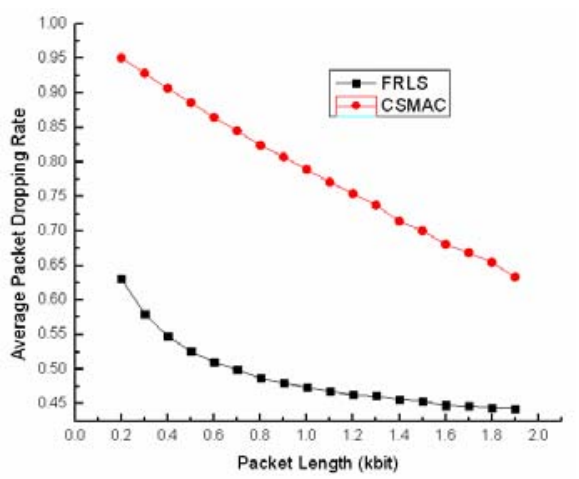

Fig. 10. Average packet dropping rate with different $L_{p k t}$.

\section{REFERENCES}

[1] I. F. Akyildiz, J. McNair, L. C. Martorell, R. Puigjaner, and Y. Yesha, "Medium access control protocols for multimedia traffic in wireless networks," IEEE Network, vol. 13, no. 4, July/Aug. 1999, pp. 39-47.

[2] N. Abramson, "The ALOHA system-Another alternative for computer communication," in 1970 Fall Joint Comput. Conf., AFIPS Conf. Proc., vol. 37, 1970, pp. 281-285 (AFIPS Press).

[3] J. Jubin and J. D. Tornow, "The DARPA packet radio network protocols," Proc. IEEE, vol. 75, no. 1, Jan. 1987, pp. 21-32.

[4] Military Standard - Interoperability Standard for Digital Message Transfer Device Subsystems, MIL-STD-188-220B, Jan. 1998.

[5] G. Anastasi, L. Lenzini, E. Mingozzi, A. Hettich, and A. Krämling, "MAC protocols for wideband wireless local access: Evolution toward wireless ATM," IEEE Personal Commun., vol. 5, Oct. 1998, pp. 53-64.

[6] Shunyuan Ye, Thanasis Korakis, Shivendra Panwar, " CSMAC: A New Centralized Scheduling-based MAC Protocol for Wireless LAN", Proc. IEEE WCNC 2009, Budapest, Hungary, Mar. 2009, pp. 1-6.

[7] Mohamed K.Watfa, OmarMirza, JadKawtharani, "BARC: A Battery Aware Reliable Clustering algorithm for sensor networks", Journal of Network and Computer Applications, vol. 32, Nov. 2009, pp. $1183-1193$ 